\title{
Starburst and Poststarburst Galaxies from the Sloan Digital Sky Survey
}

\author{
Anna Blomqvist and Nils Bergvall \\ Department of Astronomy \& Space Physics, Uppsala University, Sweden
}

\begin{abstract}
We have used the SDSS DR 4 to study links between starburst and poststarburst galaxies in the redshift range $\mathrm{z}=0-0.25$. Starburst counterparts to the most luminous postbursts seem to be lacking, probably due to an increasing contribution from dust and AGNs. Starburst lifetimes were derived from spectral evolutionary models and space densities and were found to vary with luminosity, from a few times $10^{7}$ to the order of Gyr at the low luminosity end.
\end{abstract}

Keywords. galaxies: evolution - photometry - starburst

\section{Background and results}

The SDSS samples were selected on the Balmer emission/absorption line strengths. Thus $W(H \alpha)_{e m}>100 \AA$ defined a starburst and $W(H \gamma, \delta)_{a b s}>5 \AA$ and $W(H \beta)_{a b s}>$ $2.5 \AA$ a postburst. AGNs were removed from the starburst but not from the postburst sample. The brightest poststarbursts are $\sim 1^{m}$ brighter than the starbursts, in conflict with models predicting a luminosity fading after the burst. They are also redder, have higher surface brightnesses and seem to be more nucleated. Plausible explanations are (1) the lifetime of the starburst phase of the brightest galaxies is short (2) luminous starbursts are dimmed by dust and (3) bright poststarbursts originate from starbursts contaminated by AGNs (at least $9 \%$ with $M_{r}<-20$ currently host AGNs.). Using SEMs ${ }^{[2][3]}$ and the luminosity functions (Fig. 1) we find that the starburst length increases with decreasing luminosity, from a few times $10^{7}$ yrs to $\sim$ Gyrs. At low luminosities the burst length may become of the order of a Hubble time, i.e. they hardly qualify for the epithet starburst.

Figure 1. Luminosity functions of: starburst galaxies (squares) with corrections for internal extinction (dotted line, squares), AGN dominated galaxies separated from the starburst sample (circles) and poststarburst galaxies (triangles). The dashed line show all types of galaxies ${ }^{[1]}$

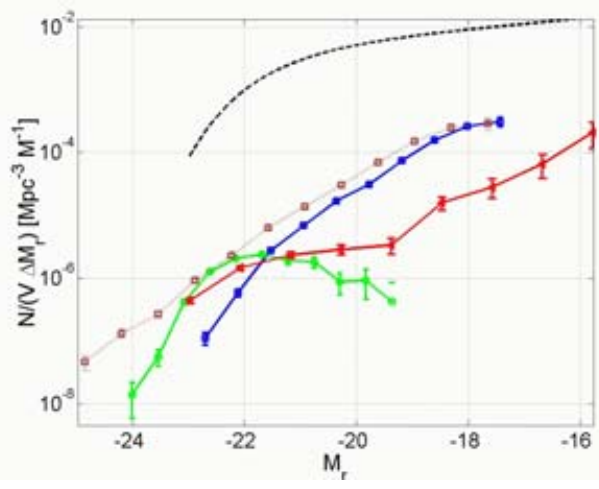

\section{References}

[1] Blanton, M. et al. 2003, AJ 125, 2348.

[2] Bruzual, G. \& Charlot, S. 2003, MNRAS 344, 1000.

[3] Zackrisson, E. et al. 2001, A $\mathscr{E} A$ 375, 814. 